\title{
Multi-messenger astroparticle physics for the public via the astroparticle.online project
}

\author{
Victoria Tokareva, ${ }^{a, *}$ Yulia Kazarina, ${ }^{b}$ Andreas Haungs, ${ }^{a}$ Dmitriy Kostunin, ${ }^{c}$ \\ Alexander Kryukov, ${ }^{d}$ Evgeny Postnikov, ${ }^{d}$ Vladimir Samoliga, ${ }^{b}$ Alexey Shigarov, ${ }^{e, f}$ \\ Dmitry Shipilov $^{g}$ and Dmitry Zhurov ${ }^{b}$ \\ ${ }^{a}$ Karlsruhe Institute of Technology, Institute for Astroparticle Physics, 76021 Karlsruhe, Germany \\ ${ }^{b}$ Irkutsk State University, Applied Physics Institute, Irkutsk 665004, Russia \\ ${ }^{c}$ DESY, 15738 Zeuthen, Germany \\ ${ }^{d}$ Lomonosov Moscow State University, SINP, Moscow 119991, Russia \\ ${ }^{e}$ Matrosov Inst. f. System Dynamics and Control Theory, Irkutsk 665044, Russia \\ ${ }^{f}$ Irkutsk State University, Irkutsk 665004, Russia \\ ${ }^{g}$ X5 Retail Group, Moscow \\ E-mail: victoria.tokareva@kit.edu
}

Multi-messenger astroparticle physics is still a young field of research and is hardly covered in educational curricula or outreach. The astroparticle.online project, founded in 2018 within the framework of the German-Russian Astroparticle Data Life Cycle Initiative (GRADLCI), encompasses an endeavor to address this issue. Within the project, scientists from Karlsruhe Institute of Technology (KIT), Irkutsk State University (ISU) and Moscow State University (MSU) developed a range of educational materials: articles, video lectures, tests, problems to solve, laboratory works and pre-trained neural networks for particle recognition. The project is supported by the KASCADE Cosmic-ray Data Center (KCDC) and GRADLCI data aggregation platform, where one can retrieve and analyze open scientific data from various experiments.

The main audience of the project's activities are high school and undergraduate students. All the educational materials are available online at the project's web portal https://astroparticle . online, they are used both in online and offline masterclasses organized by the project members, and also as the supplementary content by educational organizations: for example, in the ISU course "Introduction to experimental methods in high energy astrophysics". Over the time the project has been operating, more than 150 students took part in its activities.

This contribution will cover the experience gained while running the project for more than 3 years, our challenges and developments.

$37^{\text {th }}$ International Cosmic Ray Conference (ICRC 2021)

July 12 th - 23rd, 2021

Online - Berlin, Germany

\footnotetext{
*Presenter
} 


\section{Motivation}

Since their discovery in 1912, cosmic rays (CRs) have been studied quite thoroughly. Nevertheless, many related mysteries, such as the spectrum and mass composition of CRs, the mechanisms of CR acceleration and propagation, as well as their origin, remain the subject of active research by specialists in the field of astroparticle physics [1]. Of particular interest is the study of the behavior of matter at ultra-high energies (from $10^{18} \mathrm{eV}$ to $10^{21} \mathrm{eV}$ ) [2], which are unattainable in terrestrial accelerators, such as the Large Hadron Collider [3], at the present stage of scientific and technological progress.

One of the topical trends in this field, rapidly gaining popularity since 2017, is joint analysis of data from various experiments, carried out within the framework of multi-messenger astronomya field of science that studies data from messengers like electromagnetic radiation, gravitational waves, and elementary particles such as neutrinos and high-energy cosmic rays, in an integrated manner, in order to obtain information about the processes occurring in space [4, 5]. Joint analysis of data from different sources allows to obtain scientific knowledge that one cannot get by studying these sources separately. A good example is the combined analysis of a neutron star merger event using data from the gravitational wave signal GW170817, the short gamma-ray burst GRB170817A, and the optical source SSS17a, that shed light on the origin of heavy elements in the universe [6].

As multi-messenger astronomy is a relatively young field of research, it is very important to promote it in order to attract new personnel, to inform society about the latest scientific achievements, and to develop open science.

While a fairly high number of large educational projects such as Cosmic@Web [7], HiSPARC [8], QuarkNet [9], EEE [10], CREDO [11], and others are engaged in the development of educational content on the physics of cosmic rays, as well as very interesting examples of outreach content presented by such collaborations as LIGO-Virgo [12], IceCube [13]), etc., the popularization of multi-messenger astronomy is still in the stage of development, and interesting ways of presenting the material are yet to be found in this area.

In order to fill this gap, the astroparticle.online project was created in 2018 as an educational branch of the GRADLC [14] initiative. Collaboration in this area was supervised by the staff of the Baikal Multimessenger Lab [15] in ISU, with the support of IAP KIT, ISDCT, and MSU, as well as the participants of the TAIGA and KASCADE collaborations.

The purposes of creation included:

- Training new personnel for astroparticle physics;

- Popularization of astroparticle physics and multi-messenger astronomy;

- Informing the public about the current state of research in the field of astroparticle physics;

- Search for new methodologies for teaching the latest technologies, such as machine learning and multi-messenger analysis.

The following tasks were set to achieve these goals:

- Development and support of the astroparticle.online web portal;

- Preparation of educational materials for the portal, including interactive ones and those using machine learning;

- Organizing and carrying out educational events online and offline, applying practices obtained within the framework of the project in training courses taught at the ISU Physics Department. 
In this article, we share our experience in solving these problems. The article consists of the following parts. The introduction explains the motivation of the project, its goals and objectives. The Web portal development part describes our experience in developing the web portal, the difficulties encountered and the technical solutions that we undertook in response to them. Next, we describe the educational content of the portal in the Educational materials part, and then talk about the experience of using it in the educational environment in the Events part. Finally, in the Discussion part we briefly discuss the implications of the results obtained and summarize them in the Conclusion. The Acknowledgments part contains our thanks to colleagues who contributed valuable ideas to the development of the project.

\section{Web portal development}

The first version of the site was created in spring 2018 on the basis of the cloud infrastructure of the computing center "Integrated Information and Computing Network of the Irkutsk Scientific and Educational Complex" (IIVS IRNOK) [16]. The content management was based on an open source software platform HUBZero, which was aimed to made to host analytical tools, publish data, share resources, collaborate and build communities in a single web-based ecosystem with a focus on being used by professional researches [17].

However, during trial at the ISSAP Baikal Summer School-2018 (see the Events section), this content management system turned out to be insufficiently flexible to meet the needs of the project. In this regard, starting from 2019, astroparticle.online switched to the WordPress [18] platform, that is an open source platform developed since 2003 with a good documentation and a large user community. This CMS has been chosen among other popular content management systems like Joomla and Drupal for being superior in terms of file sizes, requests sent to server, and page load time [19].

Besides, the hosting location moved to server of ISDCT [20], while some special applications were hosted using computational resources of SINP MSU[21].

While working on the resource and communicating with its audience at educational events (see the "Events" part), we found out that the users prefer interactive content (applications, tests, tasks); however, they are more inclined to ask questions and express wishes personally during offline events rather than to write them online on the site. Thus, we decided to close the "Forum" section on the site. At the same time, we noticed interest of the public to topical news related to astroparticle physics and multi-messenger astronomy, and, in particular, to information about experiments participating in the collaboration. In this regard, when switching to a new content management platform, we introduced new sections "News" and "Experiments" to the site, respectively.

For more details about the content of the site, see sections 3-4 of the article.

The current interface of the astroparticle.online site is shown in Fig. 1.

\section{Events}

Educational materials were developed and optimized directly in contact with the portal's main audience: senior and junior university students. Thus, the Online School section of the site originally emerged as a section for publishing materials from the "Introduction to Astroparticle 


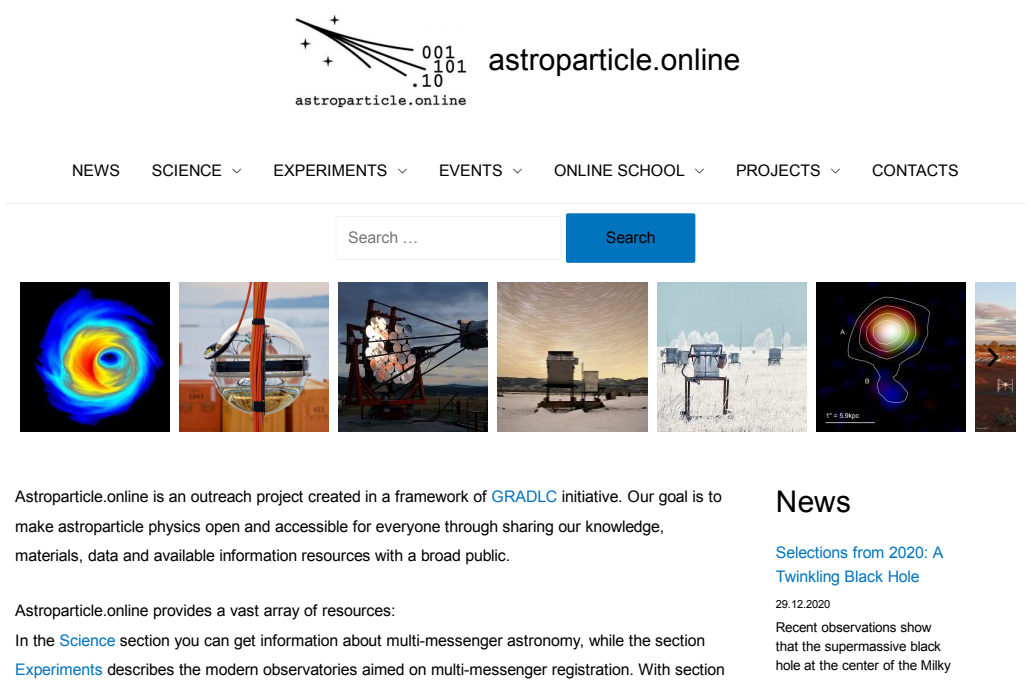

Figure 1: Screenshot of the main page of the current version of astroparticle.online web portal.

Physics" course launched at ISU in 2019 with the direct involvement of some of the authors of this article ${ }^{1}$. These materials are currently contained in the sections Lectures, Seminars, Labs and Tasks, and the course itself was further expanded with the use of the portal materials developed later. A total of 18 students have completed this course since 2019.

Collaborations with ISSAP Baikal Summer School in 2018 and 2019 had a huge impact on the project, it inspired the authors of the astroparticle.online project to prepare lectures and seminars. Their videos were later published in the relevant sections of the site. More than 150 summer school students took part in the activities offered by astroparticle.online participants.

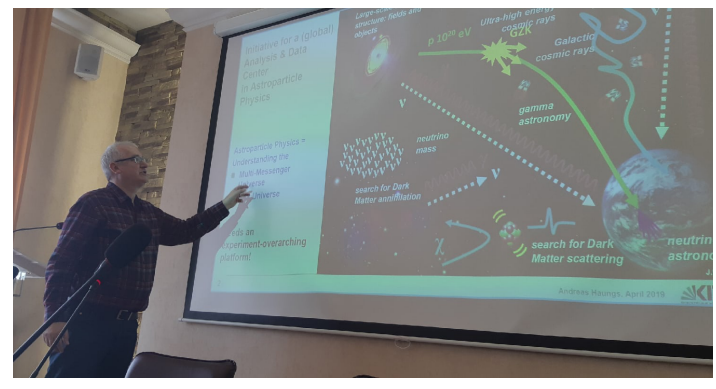

a)

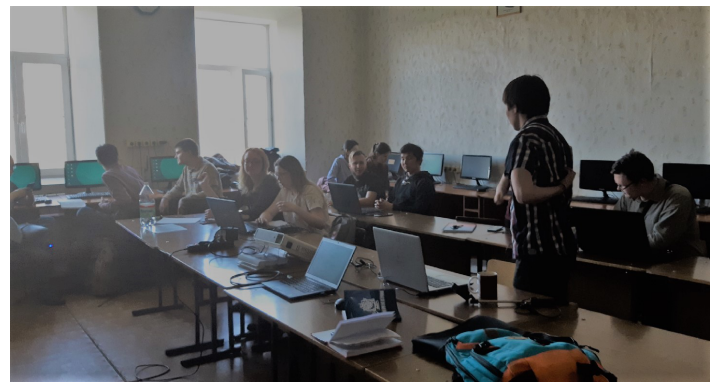

b)

Figure 2: a) Dr. A. Haungs reads a lecture on multi-messenger astroparticle physics, ISU, DLC-2019; b) ISU students are analyzing open data as part of an event organized by the astroparticle.online portal.

In addition, DLC conferences were held in 2018, 2019 and 2020 with the support of astroparticle.online, where we actively encouraged the participation of interested project users.

1https://hg.sr.ht/ kostunin/ap-seminar-latex 
Also, over the years of the project's activity, special events were held at the Days of Science of ISU, and visiting lectures of astrophysicists were carried out at the physics departement of ISU and in the planetarium of the city of Irkutsk, in particular, with international participation (see Fig. 2).

According to our estimates, about 300 people took part in our events during the activity of the project. The way they understood the material and the questions they asked had a significant impact on improving the quality of the content on the site. In addition to the original content, the site also published popular science videos from open sources and links to news publications with an indication of authorship.

At the beginning of 2020, due to well-known circumstances, the organization of massive offline events turned out to be difficult, and we are focusing our efforts on the development of online educational materials, such as tests and interactive materials based on the advances of the GRADLCI project.

\section{Online content}

\subsection{Lectures, Seminars and Tasks}

The key educational materials presented on the site are given in such traditional form as lectures, seminars and tasks for self study.

The "Lections" and "Seminars" sections present lectures and seminars based on current video materials and relevant presentations, authored by leading astrophysicists-members of the TAIGA collaboration. After each video, an online test is presented to check the knowledge gained after watching. The course workshops focus on applied knowledge of data modeling and analysis. The seminars provide an introduction to the main cosmic ray modeling software packages, namely CORSIKA, COAST, CoREAS, as well as to the main programming languages such as $\mathrm{C} / \mathrm{C}++$ and Python, and to the basic data analysis frameworks such as ROOT, numpy, scipy and matplotlib. As a result of this course, students will be able to solve problems in the field of modern analysis of astrophysical data.

A more popular presentation of information is also found in the "Popular science" section of the portal. To test knowledge on the materials of this section, special tests have been developed covering the following topics: cosmic microwave background, black holes, neutron stars, future of the universe, red dwarfs, white dwarfs.

The listed sections together represent a course of problems in astrophysics, consisting of materials and methods of testing knowledge in the form of tests or theoretical problems. The average score for the tests on the site is $74 \%$.

\subsection{Data Analysis}

In this section, users can get access to astropartilce physics data centers GRADLCI [22] and ADDPS [23]. Further, in the "Labs" section, a laboratory work is presented, designed specifically to familiarize students with astrophysical detectors, namely a laboratory telescope for studying secondary cosmic radiation. The purpose of the laboratory work is to study the main properties of secondary cosmic radiation at the observation level. The site contains a theoretical introduction, a description of the experimental setup, a measurement technique, a description of the data format 
and the processing of experimental data. In addition, experimental data from one of the runs of a laboratory telescope have been made publicly available, and can be used by users to solve two astrophysical problems: measure the intensity of secondary cosmic radiation and determine the angular distribution of secondary cosmic radiation at the observation level. Users can get the reliability of the results by comparing their results with the theoretical predictions presented in the theoretical description of the work.

For users to get familiar with the use of machine learning for the analysis of astrophysical data, the authors developed a prototype application for the Gamma/Hadron Separation in the IACT telescope (using the example of TAIGA IACT telescopes), shown in Fig. 3. This application is based on results of the study [24].

Let's check yourself and determine which type of the particle is it?

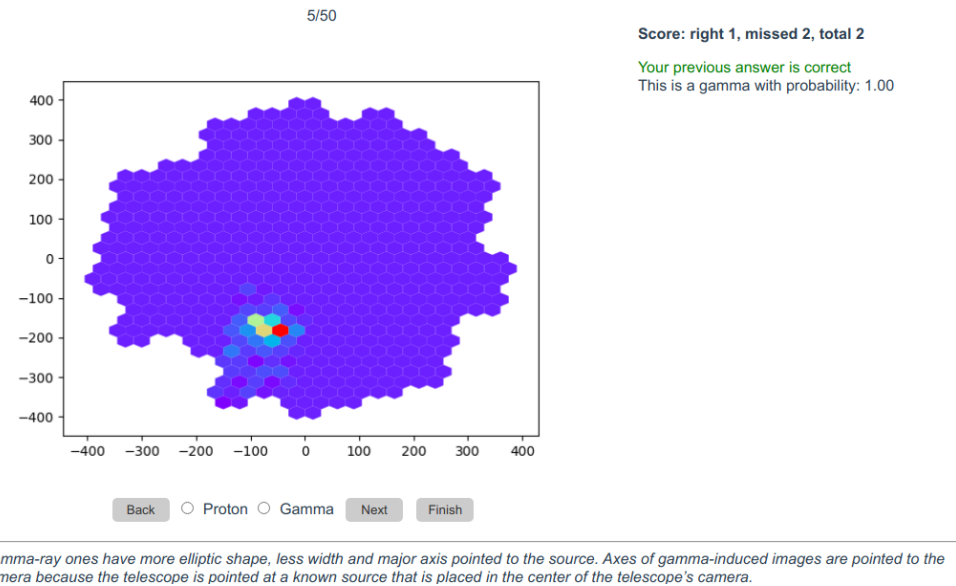

center of the camera because the telescope is pointed at a known source that is placed in the center of the telescope's camera.

Figure 3: An interface of the gamma/hadron separation application.

The service invites users to compete with a convolutional neural network (CNN) on determining a particle's type by its imprint on the IACT detector. An image of the detector response is shown to the users, and they are asked to make a suggestion about the type of the particle detected. After the answer is provided, the application shows the the CNN's decision and the correct answer. If the user's guess was correct, they get +1 point. Although so far there are no humans that have won this game against the CNN, it has been noticed that with an increase in attempts, the quality of users' answers is improving.

The software implementation of this service is using only open technologies. To this end, Django framework and Python programming language were used to develop the application, SQLite database was used for data storage, and the deployment was done employing Docker. The CNN used was implemented using the pyTorch framework, and trained on simulations of gamma and proton events for the TAIGA IACT experiment.

\section{Conclusion}

The capabilities of the educational and information portal astroparticle.online were used to attract more students to astrophysics and particle physics and to train highly qualified specialists in the field of data processing for astrophysical experiments. 
The task of preparing educational materials in the field of multi-messenger astronomy is not trivial, and requires the joint collaboration of a large number of specialists with different levels of expertise. We were one of the first educational initiatives that took up efforts in this direction, and we hope that our results, although they cannot be an unambiguous answer to the question "How to popularize multi-messenger astronomy for the general public", represent significant first steps in this direction.

\section{Acknowledgments}

The authors would like to express gratitude to the colleagues from KCDC team for sharing their expertise in data curation and teaching experience.

\section{References}

[1] T.K. Gaisser, R. Engel and E. Resconi, Cosmic rays and particle physics, Cambridge University Press (2016).

[2] M.S. Longair, High energy astrophysics, Cambridge university press (2010).

[3] "Facts and figures about the LHC." https://home. cern/resources/faqs/facts-and-figures-about-lhc.

[4] I. Bartos and M. Kowalski, Multimessenger astronomy, in Gamma-Ray Bursts, IOP Publishing (2017).

[5] E. Burns, A. Tohuvavohu, J. Bellovary, E. Blaufuss, T. Brandt, S. Buson et al., Opportunities for multimessenger astronomy in the 2020s, arXiv preprint arXiv:1903.04461 (2019) .

[6] M.R. Drout et al., Light curves of the neutron star merger GW170817/SSS17a: Implications for r-process nucleosynthesis, Science 358 (2017) 1570.

[7] “Cosmic@Web - Tools zur Online-Analyse.”https://physik-begreifen-zeuthen . desy.de/angebote/kosmische_teilchen/cosmicweb/index_ger.html.

[8] "HiSPARC." https://www.hisparc.nl/en/.

[9] “Cosmic Ray e-Lab.” https://www.i2u2.org/elab/cosmic/home/project.jsp.

[10] "Extreme Energy Events (EEE)—Science in Schools." https://eee. centrofermi .it/.

[11] P. Homola et al., Cosmic-ray extremely distributed observatory, Symmetry 12 (2020) .

[12] "Gravitational Wave Open Science Center." https://www.gw-openscience.org/interactive/.

[13] "IceCube Outreach Resources." https://icecube.wisc.edu/outreach. 
[14] V. Tokareva, I. Bychkov, A. Demichev, J. Dubenskaya, O. Fedorov, A. Haungs et al., German-Russian Astroparticle Data Life Cycle Initiative to foster Big Data infrastructure for multi-messenger astronomy, in 37th International Cosmic Ray Conference (ICRC 2021), Online, 12.07. 2021-23.07. 2021, 2021.

[15] P. Bezyazeekov, I. Bychkov, N. Budnev, D. Chernykh, Y. Kazarina, D. Kostunin et al., Towards the Baikal Open Laboratory in astroparticle physics, arXiv preprint arXiv:1906.10594 (2019) .

[16] "Integrated information and computing network of the Irkutsk research and educational complex." http://net.icc.ru.

[17] M. McLennan and R. Kennell, Hubzero: A platform for dissemination and collaboration in computational science and engineering, Computing in Science Engineering 12 (2010) 48.

[18] B. Williams, D. Damstra and H. Stern, Professional WordPress: design and development, John Wiley \& Sons (2015).

[19] S.K. Patel, V.R. Rathod and S. Parikh, Joomla, Drupal and WordPress-a statistical comparison of open source CMS, in 3rd International Conference on Trendz in Information Sciences \& Computing (TISC2011), pp. 182-187, IEEE, 2011.

[20] "Matrosov Institute for System Dynamics and Control Theory of Siberian Branch of Russian Academy of Sciences, official website." http://idstu.irk.ru/en.

[21] "Skobeltsyn Institute Of Nuclear Physics Lomonosov Moscow State University, official website." http://www. sinp.msu.ru/en.

[22] V. Tokareva, A. Haungs, D. Kang, F. Polgart, D. Wochele and J. Wochele, Data aggregation platform for experiments of astroparticle physics, in DLC 2020: Proceedings of the 4th International Workshop on Data Life Cycle in Physics. Ed.: A. Kryukov, p. 134, 2020.

[23] A. Kryukov, I. Bychkov, E. Korosteleva, A. Mikhailov and M.-D. Nguyen, AstroDS-A distributed storage for astrophysics of cosmic rays. Current status, arXiv preprint arXiv:2010.04938 (2020) .

[24] E.B. Postnikov, A.P. Kryukov, S.P. Polyakov, D.A. Shipilov and D.P. Zhurov, Gamma/hadron separation in imaging air cherenkov telescopes using deep learning libraries tensorflow and pytorch, in Journal of Physics: Conference Series, vol. 1181, p. 012048, IOP Publishing, 2019. 